\title{
The ascending branch of the lateral circumflex femoral artery penetrating the posterior division of the femoral nerve
}

\author{
Hankyu Kim ${ }^{1,2}$, Yong Seok Nam ${ }^{1,2}$, Yi-Suk Kim ${ }^{1,2}$ \\ ${ }^{1}$ Department of Anatomy, College of Medicine, The Catholic University of Korea, Seoul, ${ }^{2}$ The Catholic Institute for Applied Anatomy, College of \\ Medicine, The Catholic University of Korea, Seoul, Korea
}

\begin{abstract}
Understanding anatomic variations in neurovascular structure inside the femoral triangle is crucial for regional anesthesiologists performing femoral nerve block. During routine dissection of a cadaver, an ascending branch of the lateral circumflex femoral artery with an anomalous course passing through the femoral nerve, specifically the posterior division, was identified inside the femoral triangle on the left thigh. The novel variation identified in this study occurred in an early stage of prenatal development. Recognition of this anatomic variation will be helpful for reducing unexpected complications during the femoral nerve block and the tensor fascia latae flap. Penetration of the posterior division of the femoral nerve by the arterial branch might cause pain or paresthesia of the medial aspect of the leg in the distribution of the saphenous nerve.
\end{abstract}

Key words: Femoral nerve, Intraneural vessel, Lateral circumflex femoral artery, Anatomic variation

Received September 3, 2020; Revised October 23, 2020; Accepted November 10, 2020

\section{Introduction}

The femoral nerve arises from the dorsal branch of the second to fourth lumbar ventral rami. This nerve, after passing behind the inguinal ligament into the thigh, is separated into anterior and posterior divisions by the lateral circumflex femoral artery. The branches of the anterior division are the medial and the intermediate cutaneous femoral nerve and the nerve to the sartorius. Meanwhile, the posterior division is composed of the saphenous nerve and the nerves to the quadriceps femoris, which are related to the sensory profile of the medial aspect of the leg and extension of the knee joint, respectively [1].

\footnotetext{
Corresponding author:

Yi-Suk Kim (10)

The Catholic Institute for Applied Anatomy, College of Medicine, The Catholic University of Korea, 222 Banpo-daero, Seocho-gu, Seoul 06591, Korea

E-mail:jwkiss@catholic.ac.kr
}

The lateral circumflex femoral artery, mostly branching from the root of the deep femoral artery, passes between divisions of the femoral nerve. This artery then divides into the ascending, transverse, and descending branches. The ascending branch courses superiorly behind the tensor fasciae latae muscle to the hip joint and supplies the greater trochanter, the femoral neck, and the femoral head with its anastomosing arteries [1].

Recognition of the normal anatomy and understanding possible variations of the neurovascular structure inside the femoral triangle are important for regional anesthesiologists performing the femoral nerve block with minimal complications [2]. Also, some variations may be associated with pathologic conditions in the lower extremities. Herein, we report a case of an ascending branch of the lateral circumflex femoral artery showing an anomalous course passing through the nervous structure inside the femoral triangle. 


\section{Case Report}

During routine dissection of the thigh, unilateral variation of the femoral nerve penetrated by the ascending branch of the lateral circumflex femoral artery was identified in an 80 -year-old Korean male cadaver. No signs of previous surgery or injury were observed in this region. On the left side, the femoral nerve was already split into the anterior and the posterior divisions at a point higher than the location of the lateral circumflex femoral artery. The lateral circumflex femoral artery branched from the deep femoral artery behind the femoral nerve and spit off into the ascending branch as usual. On its way to the hip joint, the ascending branch passed through the neighboring femoral nerve, particularly the posterior division. The distance from the inguinal ligament to the point where the ascending branch pierced the posterior division of the femoral nerve was about $8.4 \mathrm{~cm}$.

The further courses of the ascending branch and the femoral nerve were similar to those previously described. The ascending branch coursed superiorly and laterally to the hip joint behind the tensor fascia latae. Meanwhile, the posterior division of the femoral nerve was divided into the saphenous nerve and the nerves to the quadriceps femoris. The tributaries of the lateral circumflex femoral vein coursed independently from the artery, not penetrating the femoral nerve. There was no corresponding variation on the right side.

\section{Discussion}

There have been multiple reports of partial splitting and reunion of the femoral nerve at different locations. Anloague and Huijbregts [3] identified bifurcated or trifurcated femoral nerves, separated by the psoas major muscle fibers at the posterior abdominal wall, that rejoined before passing behind the inguinal ligament. Variations of the femoral nerve pierced by muscular slips from the iliacus or the psoas major in the iliac fossa have also been commented on [4]. Wong et al. [5] identified multi-split femoral nerve by a variant psoas quartus muscle. However, no cases of femoral nerve split and reunification in the femoral triangle have been reported before, to the best of our knowledge.

Anatomy of the ascending branch of the lateral circumflex femoral artery was investigated, particularly in regard to its origin, course, and distributions, for the clinical conduct of flap surgery [6]. However, to the best of our knowledge, the ascending branch of the lateral circumflex femoral artery coursing through the femoral nerve with penetration has not been described yet.

The hind-limb buds are formed in the fifth week of gestation and, during this period, muscles bring associated nerves from the torso into the limb buds [5]. This is also true in the case of the femoral nerve. The arterial system of the lower extremity begins to develop from the fifth week of gestation
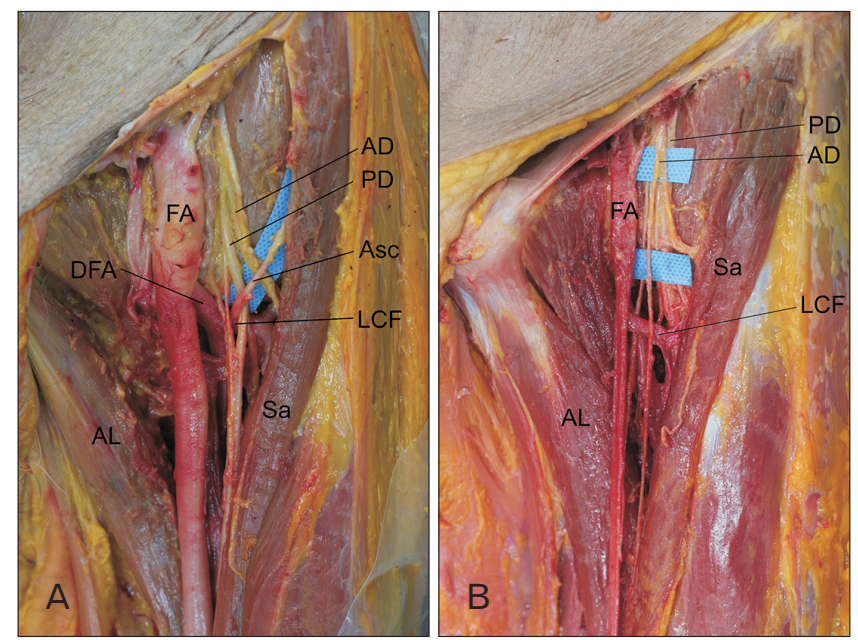

Fig. 1. (A) A case of anatomical variation in the femoral triangle of the left thigh. The ascending branch of the lateral circumflex femoral artery pierces the posterior division of the femoral nerve. The penetration site was partially dissected to expose the root of the ascending branch of the lateral circumflex femoral artery. (B) Normal anatomical structure in the femoral triangle of the left thigh. Blue cloth was inserted to make a clear distinction between the anterior division and the posterior division of the femoral nerve. AD, anterior division of the femoral nerve; $\mathrm{AL}$, adductor longus muscle; Asc, ascending branch of the lateral circumflex femoral artery; DFA, deep femoral artery; FA, femoral artery; LCF, lateral circumflex femoral artery; PD, posterior division of the femoral nerve; Sa, sartorius muscle. 
and is completed in the third month with a pattern corresponding to that of a typical adult $[7,8]$. Both the sciatic artery and the external iliac artery, during the embryogenic development, develop from the umbilical artery and form the rete femorale together, which supplies the lower-limb bud [8]. The femoral artery and the deep femoral arteries develop from the rete femorale with regression of the sciatic artery [8]. Senior [8] previously described the origin of the circumflex arteries from the rete femorale, whereas Ciftcioglu et al. [9] presumed the circumflex branches of the deep femoral artery might develop independently from the abovementioned rete femorale depending on their blood-flow distribution territory. Regarding these facts, variation in the femoral nerve and the lateral circumflex femoral artery of the type observed in this study occurs during the early stage of prenatal development.

Pathologic conditions with penetration of a nerve by a neighboring vessel have been identified at different locations. Patrick [10] reported a case of tic douloureux caused by the anterior inferior cerebellar artery passing through the trigeminal nerve. In rare cases of trigeminal neuralgia, intraneural vessels penetrating the trigeminal nerve have been identified $[11,12]$. Hemifacial spasm patients whose symptoms were caused by intraneural vessels passing through the facial nerve were also reported [13, 14]. Considering the abovementioned cases in which the intraneural vessels resulted in neuropathies, penetration of the posterior division of the femoral nerve by the arterial branch might also have caused pain or paresthesia, particularly of the medial aspect of the leg in the distribution of the saphenous nerve derived from the posterior division. However, it was not possible to identify the preterm pathologies of the donor used in this study because of the limited available medical records.

Awareness of this anatomic variation is important for regional anesthesiologists performing the femoral nerve block [2]. The inguinal crease has been recommended for transducer placement or needle insertion site in ultrasoundguided regional anesthesia for the femoral nerve block [15]. According to Lechner et al. [16], the inguinal creases lies distal to the inguinal ligament by about $6.7 \mathrm{~cm}$. Considering that the point where the ascending branch of the lateral circumflex femoral artery pierced the posterior division of the femoral nerve was $8.4 \mathrm{~cm}$ below the inguinal ligament in this case, this point was located near the inguinal crease. Therefore, displacement of the needle into the arterial branch could happen due to the intimate anatomical rela- tionship between the femoral nerve and the arterial branch of this case, which can cause unexpected complications such as hematoma formation or local anesthetic systemic toxicity [17]. Conducting ultrasound visualization with recognition of possible vascular variations could reduce the complication rate [2].

Flap surgeons should also consider this possible variation when harvesting the arterial pedicle for the tensor fascia latae flap. The ascending branch of the lateral circumflex femoral artery forms the major pedicle of the tensor fasciae latae musculocutaneous flap [6]. In a patient with this variation, the risk of accidental nerve injury would increase while approaching the arterial pedicle because of the intimate anatomical relationship between the ascending branch of the lateral circumflex femoral artery and the femoral nerve.

This is solely based on anatomical dissection, thus the information of depth dimension is lacked. Also, frequency of the variation cannot be suggested.

Despite the abovementioned limitations, an understanding of the anatomic variation identified in this study will be helpful for regional anesthesiologists to minimize unexpected serious complications during the femoral nerve block. In addition, the intraneural vessels identified in the femoral triangle may pose possible pathogeneses of refractory leg pain.

\section{ORCID}

Hankyu Kim: https://orcid.org/0000-0002-9378-6502

Yong Seok Nam: https://orcid.org/0000-0001-8698-639X

Yi-Suk Kim: https://orcid.org/0000-0003-4793-8875

\section{Author Contributions}

Conceptualization: HK. Data acquisition: HK. Data analysis or interpretation: HK, YSN, YK. Drafting of the manuscript: HK. Critical revision of the manuscript: YSN, YSK. Approval of the final version of the manuscript: all authors.

\section{Conflicts of Interest}

No potential conflict of interest relevant to this article was reported.

\section{Acknowledgements}

This research was supported by the Basic Science Re- 
search Program through the National Research Foundation of Korea (NRF) funded by the Ministry of Education (2018R1D1A1B07048476).

\section{References}

1. Standring S, Gray H. Gray's anatomy: the anatomical basis of clinical practice. 41st ed. Philadelphia: Elsevier; 2016.

2. Ishiguro S, Yokochi A, Yoshioka K, Asano N, Deguchi A, Iwasaki Y, Sudo A, Maruyama K. Technical communication: anatomy and clinical implications of ultrasound-guided selective femoral nerve block. Anesth Analg 2012;115:1467-70.

3. Anloague PA, Huijbregts P. Anatomical variations of the lumbar plexus: a descriptive anatomy study with proposed clinical implications. J Man Manip Ther 2009;17:e107-14.

4. Unat F, Sirinturk S, Cagimni P, Pinar Y, Govsa F, Nteli Chatzioglou G. Macroscopic observations of muscular bundles of accessory iliopsoas muscle as the cause of femoral nerve compression. J Orthop 2018;16:64-8.

5. Wong TL, Kikuta S, Iwanaga J, Tubbs RS. A multiply split femoral nerve and psoas quartus muscle. Anat Cell Biol 2019;52:208-10.

6. Saadeh FA, Haikal FA, Abdel-Hamid FA. Blood supply of the tensor fasciae latae muscle. Clin Anat 1998;11:236-8.

7. Keen JA. A study of the arterial variations in the limbs, with special reference to symmetry of vascular patterns. Am J Anat 1961;108:245-61.

8. Senior HD. An interpretation of the recorded arterial anomalies of the human pelvis and thigh. Am J Anat 1925;36:1-46.
9. Ciftcioğlu E, Kale A, Kopuz C, Edizer M, Aydin E, Demir MT. Medial circumflex femoral artery with different origin and course: a case report and review of the literature. Folia Morphol (Warsz) 2009;68:188-91.

10. Patrick BS. Unusual nerve-artery relationship in microvascular exploration for tic douloureux. Neurosurgery 2005;56:E629.

11. Helbig GM, Callahan JD, Cohen-Gadol AA. Variant intraneural vein-trigeminal nerve relationships: an observation during microvascular decompression surgery for trigeminal neuralgia. Neurosurgery 2009;65:958-61; discussion 961.

12. Zheng X, Feng B, Hong W, Zhang W, Yang M, Tang Y, Zhong J, Hua X, Li S. Management of intraneural vessels during microvascular decompression surgery for trigeminal neuralgia. World Neurosurg 2012;77:771-4.

13. Feng BH, Zheng XS, Wang XH, Ying TT, Yang M, Tang YD, Li ST. Management of vessels passing through the facial nerve in the treatment of hemifacial spasm. Acta Neurochir (Wien) 2015;157:1935-40; discussion 1940.

14. Oh CH, Shim YS, Park H, Kim EY. A case of hemifacial spasm caused by an artery passing through the facial nerve. J Korean Neurosurg Soc 2015;57:221-4.

15. Muhly WT, Orebaugh SL. Ultrasound evaluation of the anatomy of the vessels in relation to the femoral nerve at the femoral crease. Surg Radiol Anat 2011;33:491-4.

16. Lechner G, Jantsch H, Waneck R, Kretschmer G. The relationship between the common femoral artery, the inguinal crease, and the inguinal ligament: a guide to accurate angiographic puncture. Cardiovasc Intervent Radiol 1988;11:165-9.

17. Jeng CL, Torrillo TM, Rosenblatt MA. Complications of peripheral nerve blocks. Br J Anaesth 2010;105 Suppl 1:i97-107. 